\title{
Mei Xue* \\ Presenting Examples in Learners' Dictionaries to Assist Chinese Learners in Writing English Texts
}

\begin{abstract}
It has been widely acknowledged that exemplification plays a very significant role in foreign language learning. Many studies have been conducted on dictionary examples, but little attention has been paid to how examples can assist a specific group of foreign learners engaged in a specific learning activity. The present study is intended to fill this gap by investigating how to optimize examples in English learners' dictionaries to help Chinese learners with writing in English. The functional theory of lexicography will be employed in this study. This investigation will be carried out in two stages. The first one consists in identifying the lexicographic needs of foreign learners engaged in the task of English writing and consulting dictionary examples. The second stage is a demonstration of some proposals for exemplification in learners' dictionaries through model articles. The purpose of this study is to give new insights into exemplification in learners' dictionaries for future lexicographic research and practice.
\end{abstract}

\section{Introduction}

Examples have been viewed as a significant part in learners' dictionaries since the early efforts of Michael West and A.S. Hornby in the 1930s and 1940s. Examples could function as "attestation of the existence of a word and a sense, elucidating the meaning of the word, illustrating the contextual features, such as syntax, collocation, register, etc" (Atkins/Rundell 2008: 453-454). It has been widely acknowledged that exemplification plays a very significant role in facilitating foreign language learning (Creamer 1987; Cowie 1989; Laufer 1992; Rundell 1999; Humblé 2001; Xu 2006). The rise of corpora in the 1980s raised the question of the respective merits and demerits of authentic and made-up examples (Drysdale 1987; Laufer 1992; Humblé 1998; Nesi 2000; Atkins/Rundell 2008). Despite their respective research focuses, all these studies on exemplification show positive values of exemplification in foreign language learning. Laufer (1992: 71) states that "a correct and natural use of a word in a sentence, or several sentences, will necessarily bring out the grammatical, semantic, pragmatic and collocational characteristics of the word."

In spite of the ever-increasing number of studies on dictionary examples, little attention has been paid to how examples can assist a specific group of learners in a specific foreign language learning activity. Although Hausmann and Gorbahn (1989: 45) maintain that “... it is the examples, above all, which help the foreign learner to understand a word (decoding function), learn it (learning function) and use it (encoding function)", they do not specify the characteristics of examples in different contexts. Humblé (2001: 62) challenges the possibility of "one example to satisfy learners' divergent needs and serve different types of users", and focuses on exploring the criteria of exemplification intended for encoding (production) purposes. However, Humblé's study seems too ambitious in trying to discuss examples in monolingual as well as bilingual dictionaries used by learners having different language and culture backgrounds. As foreign language learning takes place in certain socio-economic contexts, foreign learners' lexicographic information needs tend to be language-specific. Xu (2006: 154-158) attempts to fill this gap by investigating Chinese EFL learners' reference needs in the light of dictionary examples. Xu's (2006: 172) study, based

\footnotetext{
* Mei Xue

Centre for Lexicography

Aarhus University, Business and Social Sciences

Fuglesangs Allé 4

DK-8210 Aarhus $V$

meix@asb.dk
} 
on data collected through questionnaires, attempts to identify the information category that Chinese EFL learners expect to extract from examples. However, the findings are rather unconvincing, as the study does not indicate the particular learning activity in which Chinese learners need the specific categories of information. It is also doubtful whether Chinese learners can explicitly define their reference needs for certain types of information without regard to the specific learning activities triggering these needs.

In contrast with the current lexicographic studies concerning exemplification in learners' dictionaries, this study takes functional theory of lexicography (Bergenholtz/Tarp 2003; Tarp 2008) as its starting point, focusing on exploring how to optimize the function of exemplification in a specific foreign language learning situation (e.g. English writing). The functional theory (Bergenholtz/Tarp 2003; Tarp 2008) holds that central attention is to be paid to dictionary users' lexicographic needs arising from particular lexicographically related situations, because the ultimate purpose of all lexicographic data in dictionaries is to fulfill the targeted dictionary users' individualized information needs. Accordingly, it is superfluous to discuss the quality and contents of examples divorced from a proper understanding of the targeted dictionary users' specific information needs in their concrete learning activities.

Chinese non-English majors at their initial stage of tertiary education will be taken as the targeted dictionary users in this study. The seventh edition of Oxford Advanced Learner's dictionaries of English (henceforth OALD7), the fourth edition of Longman Dictionary of Contemporary English (henceforth LDOCE4) and the second edition of Longman Language Activator (henceforth LLA2) will be under close examination. The first two dictionaries were chosen because they are very popular among Chinese learners of English. The last one was chosen because it is production-oriented. This explorative study takes two stages: identifying Chinese learners' lexicographic information needs from examples when they are engaged in English writing, and proposing some principles for exemplification with an attempt to assist Chinese learners' English writing to an optimal point.

\section{Identifying Chinese learners' lexicographic needs from examples}

Examples are assumed to be effective in presenting "syntactic behavior, collocational preferences and selectional restrictions, sociolinguistic features (including register and regional variety), semantic features and contextual effects" (Rundell 1999: 37). But the criteria to evaluate the efficiency of exemplification are based on the extent to which they serve foreign learners' idiosyncratic information needs in their individualized situations, rather than the pure linguistic data in examples themselves. A description of the profile of Chinese learners engaged in English writing definitely sheds light on understanding their specific lexicographic information needs when they consult examples to assist their English writing.

\subsection{The profile of Chinese learners engaged in English writing}

The functional theory (Tarp 2008: 137) proposes a framework with twelve parameters to define the profile of foreign learners when discussing their specific lexicographic needs in connection with foreign language learning. Given the research purpose and the targeted learner group of this study, the framework should be adapted accordingly when it comes to defining the profile of Chinese learners engaged in English writing. The existing research on the subject matter (Cortazzi/ Jin 1996; Yang 2001; Wang/Wen 2002; Chan 2004; Hu 2005; Huang 2005; Jin/Yang 2006; Tang 2006) reveals that Chinese learners' errors in English writing could be attributed to the contrastive grammatical or syntactic systems in English and Chinese, the didactics in China English education context, the learners' rare exposure to English contexts, and the different conceptual understanding of the world due to Chinese culture. The statistics abstracted from Chinese English learner corpus (henceforth CLEC) (Gui/Yang 2003) supply quantitative evidence for the most common errors committed by Chinese learners writing English texts. Accordingly, the most sali- 
ent variables shaping the profile of Chinese learners engaged in English writing are their mother tongue competence (L1), their English competence (L2), their learning contexts, and their world knowledge. These parameters characterize Chinese learners' particular lexicographic needs for the information they expect to abstract from examples.

The statistic data concerning the high-frequency error types will be abstracted from CLEC to supplement the understanding of the profile of Chinese learners as well as their lexicographic information needs. The data are chosen as a quantitative support in this study for the following reasons. First, CLEC is composed of English written texts produced by Chinese learners at different levels, including the secondary level, CET 4 (a national English test for non-English majors in their first or second year) level, CET 6 (a national English test for non-English majors after they pass CET4) level, TEM4 (a national English test for English majors in their sophomore year) level and TEM8 (a national English test for English majors in their senior year) level. These texts produced by Chinese students at different levels are respectively named as St2 texts, St3 texts, St4 texts, St5 texts and St6 texts. Second, data in CLEC have been error-tagged, which makes it easy to abstract the common errors committed by Chinese learners. Third, the error pattern abstracted from CLEC could shed light on understanding Chinese learners' common difficulties in English writing, as the centroid factor analysis (Gui/Yang 2003: 51) demonstrates that the errors committed by Chinese learners at different levels differ only in quantity, not in quality.

Generally, most Chinese learners (e.g. non-English majors) in the mainland of China have been studying English for at least 6 years in formal educational contexts upon reaching their undergraduate level (Wu 2001; Hu 2005). They are expected to continue learning English in classroom during the first two years at university. Their age normally ranges from 17 to 20. Despite the wide range of varieties of local dialects they speak in their hometowns, all Chinese learners speak fluent Mandarin Chinese and share the same writing (the simplified Chinese characters) and phonetic transcription system (the Romanized Pinyin). According to the English syllabus set by the Chinese Ministry of Education, these post-secondary learners are supposed to have a command of a 3,300 core vocabulary and normally be able to produce a piece of English writing about 100120 words on a given topic within 30 minutes. It should be noted that English is a foreign language consciously learned in formal educational contexts in China. Chinese learners mainly assimilate the knowledge of English vocabulary and grammar from textbooks. Otherwise their exposure to English is rare and limited. Therefore, it could be inferred that Chinese learners' skills in using English may lag behind their knowledge about English lexis and grammar. When writing English texts, if they have difficulties in articulating their ideas directly in English, they may resort to Chinese to formulate their ideas and subsequently figure out the proper lexical equivalents as well as possible sentence structures in English later. Inevitably, there is "existence of negative transfer from Chinese to English at the phonological, lexical, syntactic, semantic or discoursal level” (Chan 2004: 57). Many studies (Mohan/Lo 1985; Yang 2001; Wang/Wen 2002; Chan 2004; Huang 2005) have confirmed the interference of mother tongue in texts written by Chinese learners of English.

CLEC identifies 61 types of errors in such texts and groups them into 11 categories. The frequency rank of the 11 error categories is as follows: morphology, words, syntax, verbs, nouns, collocations, pronouns, prepositions, adjectives, adverbs, conjunctions (Gui/Yang 2003: 48). The results of the centroid factor analysis (Gui/Yang 2003: 49) indicate that all types of errors could be interpreted as related to either semantics or syntax. The distribution of the errors (see graph 4.1 in Gui/Yang 2003: 49) is as follows: morphology (26\%), words (25\%), syntax (16\%), verbs (15\%), nouns (8\%) and others (10\%). Table 1 below is intended to present the high-frequency error types related to these five major error categories on the basis of the norm-processed statistic data shown in the general table of error frequency (see table 4.2 in Gui/Yang 2003: 46-48). Considering the profile of Chinese learners in this study, only the top three error types identified in St2 and St3 texts are abstracted through token concordance. Examples are randomly extracted from the concordance results to illustrate the error types. No examples have been provided for errors 
in syntax category in table 1 , given the fact that errors in syntax must be viewed in a larger context. For the ease of data interpretation, corrections are presented in the following square brackets. It should, however, be noted that the error tags could be interpreted differently depending on research perspective. For instance, shaked is tagged as a spelling error in CLEC, but it could be interpreted as an error related to inflection as well. Further interpretations of errors will be presented in the column 'Notes'.

\begin{tabular}{|c|c|c|c|c|}
\hline Category & Token & Error type & Examples & Notes \\
\hline \multirow{3}{*}{ Morphology } & $\mathrm{fm} 1$ & Spelling & $\begin{array}{l}\text { worset[worst];suitabe[suitable]; } \\
\text { approachs[approaches] }\end{array}$ & spelling or inflection \\
\hline & fm3 & Capitalization & chinese[Chinese] & \\
\hline & fm2 & Word building & $\begin{array}{l}\text { eatting[eating]; childrens[children];rainly } \\
\text { [rainy] }\end{array}$ & inflection or derivation \\
\hline \multirow{3}{*}{ words } & wd3 & Substitution & healthy body[health];Japan[Japanese] food & $\begin{array}{l}\text { expression or word } \\
\text { class }\end{array}$ \\
\hline & wd4 & Absence & $\begin{array}{l}\text { difference[a difference]; reached[...]; can } \\
\text { [be ]sure }\end{array}$ & $\begin{array}{l}\text { article, transitivity, } \\
\text { word class }\end{array}$ \\
\hline & wd2 & Part of speech & $\begin{array}{l}\text { was happily[happy]; will success[succeed]; } \\
\text { the imply[implication] }\end{array}$ & - \\
\hline \multirow{3}{*}{ syntax } & sn8 & Structure & - & - \\
\hline & $\operatorname{sn} 9$ & Punctuations & - & - \\
\hline & sn1 & Run-on sentence & - & - \\
\hline \multirow{3}{*}{ verbs } & vp6 & Tense & It take [takes];I begin[began] & tense or agreement \\
\hline & vp3 & Agreement & diet are[is]; a person want[wants] & - \\
\hline & vp1 & Pattern/transitivity & listen [to] the music & _ \\
\hline \multirow{3}{*}{ nouns } & np6 & Number & secret[a secret]; worker[workers] & $\begin{array}{l}\text { article, countability, or } \\
\text { plural inflection }\end{array}$ \\
\hline & np3 & Agreement & $\begin{array}{l}\text { these thing[things]; four chair[chairs ]; } \\
\text { some reason[reasons ] }\end{array}$ & 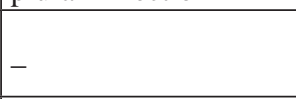 \\
\hline & np7 & Article & a [an ]important; a [an ] hour; the[ a ] way & _- \\
\hline
\end{tabular}

Table 1. High-frequency error types in texts written by Chinese learners' of English

Table 1 together with the statistic data from CLEC (for a more detailed description, see Gui/Yang 2003: 10-54) shows that Chinese learners make many errors in spelling, tenses, agreement, verb transitivity, inflections, use of articles, nominal countability, syntactic structure, and word class recognition. It is common for Chinese learners to produce utterances like *He feel very happy.; *He visit his parents last week.; *a news; *two breads; *rised; *let sb to do sth, and so on. Besides these linguistic errors, Chinese learners may also commit errors related to culture and pragmatics in English writing, due to the cultural difference between the West and the East (Yang 2002). For instance, they may address people by their job titles, such as Teacher/Engineer Zhang, dear teacher, and so on. The pragmatic formulae, such as greeting, requesting and complimenting also differ significantly in Chinese and English cultures, and tend to pose a challenge to Chinese learners' in various writing activities, such as in business letter writing.

\subsection{Lexicographic needs of Chinese learners' engaged in English writing}

On the basis of the above description of the profile of Chinese learners and their common difficulties in English writing, one can claim that Chinese learners generally consult examples for the following information: 
- Information about the patterns of syntactic behavior of vocabulary, especially those concerning syntactic structure, verb forms, verb transitivity, agreement, inflection, nominal countability, article use, collocation, and word class

- Information about the syntactic constraints of words

- Information about the contextual meaning of words

- Information about pragmatic restrictions and cultural differences

In order to actively accommodate Chinese learners' information needs, the nature and contents of these information categories must be adapted to the characterized with the profile of the Chinese learners engaged in English writing activities.

\section{The present lexicographic practice of exemplification}

It goes without saying that dictionary users appreciate examples, and that examples are helpful in foreign text production. But it is doubtful whether the present lexicographic practice of exemplification is as efficient as many studies have assumed. The purpose of examining examples in the selected entries from the chosen dictionaries is two-fold: (1) to understand to what extent the present lexicographic practice can satisfy Chinese learners' information needs and (2) to propose suggestions for possible improvements. Examples from the article suggest in the chosen dictionaries are examined below. The choice of the word suggest has been determined by the fact that this word tends to pose difficulty for Chinese learners engaged in English writing activities. Instances of the Chinese learners' productive use of suggest are abstracted from the St2 texts (14 records) and St3 texts (13 records) in CLEC. Only the erroneous uses (12 records) are presented in table 2. The mistakes in the use of suggest mainly fall into three categories: i) wrong syntactic structure (e.g. suggest to do sth, suggest sb sth, and suggest sb to do sth), ii) improper verb forms in that-clause (e.g. suggest we don't, suggest you will, and suggest that we must), and iii) inappropriate lexical choice in terms of meaning (e.g Deng Xiao-ping suggested the reform).

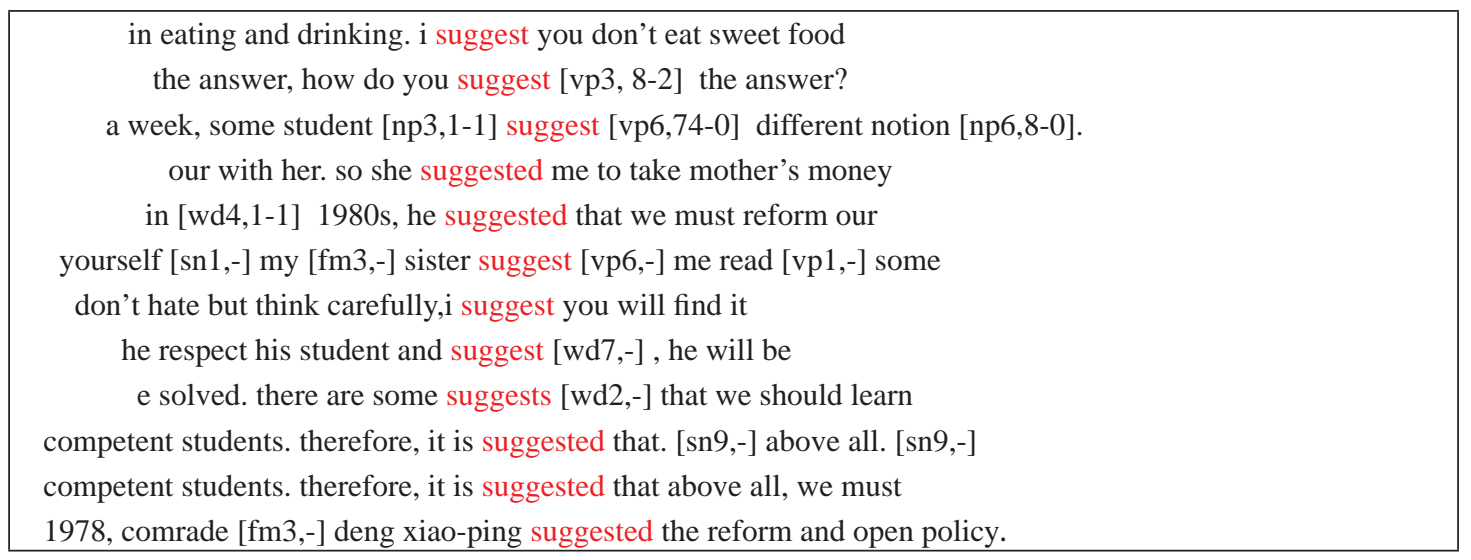

Table 2. Chinese learners' inappropriate use of suggest as shown in CLEC

The following section will examine whether the chosen dictionaries could help Chinese learners tackle their difficulty with suggest. Tables 3, 4 and 5 show the examples in the entry suggest extracted from the chosen dictionaries. For the ease of reference, these examples are numbered in accordance with the order of the sub-senses in dictionary entries. If there is more than one example under the same sub-sense, the examples will be numbered alphabetically. For instance, $1 a$ and $1 b$ indicate that examples $a$ and $b$ belong to the same sub-sense 1 in the entry. The typographic features shown in dictionary entries are maintained in the tables. 


\subsection{Deficiency in the examples in OALD7}

As shown in Table 3, sixteen examples are presented in the entry suggest in $O A L D 7$ according to their different contextual meanings. Syntactic structures, such as suggest sth (to sb), and suggest sb/sth (for sth) are indicated as signposts before sentence examples.

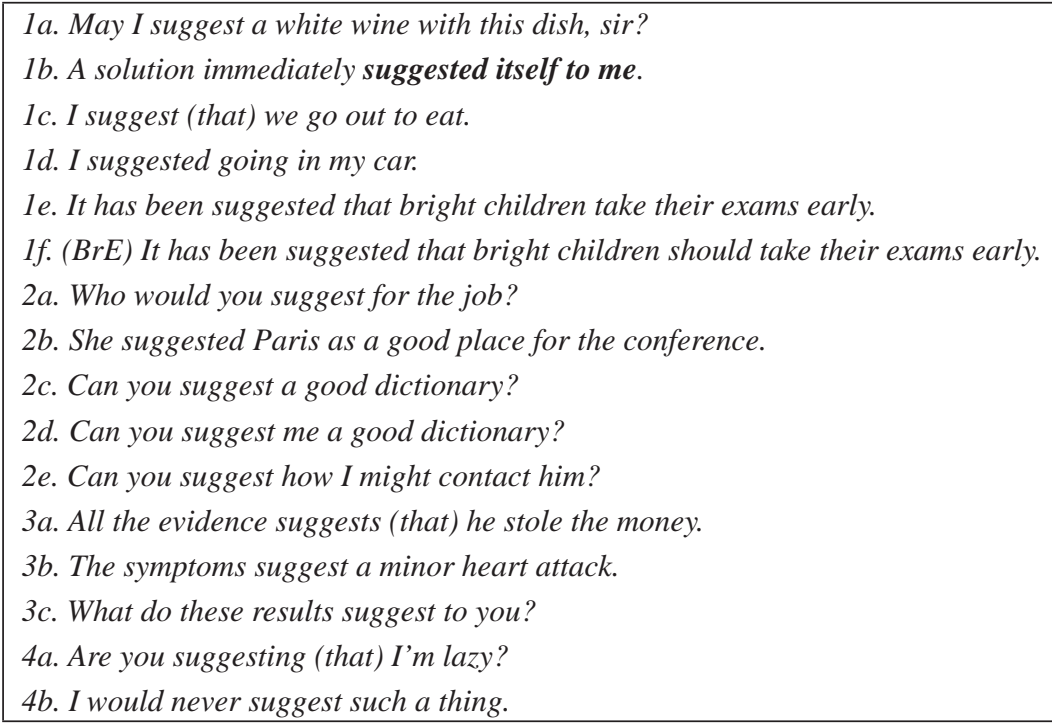

Table 3. Examples from OALD7

\subsubsection{The implicit indication of the syntactic structure}

Considering the fact that Chinese learners using the word suggest in text production tend to make errors in syntactic structure, it would be helpful to present or indicate, in one way or another, the typical syntactic structure in which the word appears. The syntactic structure encoded in examples $2 b$ and $2 c$ may be self-evident to other group of foreign learners, but it is not evident for Chinese learners. Similarly, the syntactic structure (using the grammatical code $\boldsymbol{w} \boldsymbol{h}$ with the example $2 e$ ) may be obvious for native speakers, but Chinese learners who are still in the process of developing their English literacy may be unconscious or uncertain of the possible constructions, such as how, where, etc. Such examples cannot prevent Chinese learners from producing sentences like He suggested to go by plane. He suggested us to leave earlier.

\subsubsection{The inefficient indication of grammatical constraints}

Examples $1 c, 1 e$ and $1 f$ are intended to indicate the syntactic structures suggest + that and It has been suggested that .... The connective that should not be omitted when the that-clause is in the passive voice. Moreover, when suggest acquires the contextual meaning equal to propose and precedes a that-clause, the verb in the that-clause should take the canonical form regardless of the tenses in the main clause. Table 3 shows that Chinese learners of English tend to make errors in this respect. However, this important syntactic message seems to be missing from the examples. It could be argued that such message is encoded in examples $1 e$ and $1 f$, but it is rather challenging for Chinese learners to abstract these grammatical constraints. Furthermore, it is very doubtful that Chinese learners could extract the constraints of the syntactic pattern suggest sb sth from example 2, as there are no corresponding grammatical constraints in Chinese syntactic environments. Sentence examples as the following will help Chinese learners in the process of text production in a real sense: He suggested we (should) go out to eat. He suggested that the lecture (should) be given in English. In short, explicitly indicating the possible grammatical constraints 
could reduce the Chinese learners' cognitive efforts in processing the relevant data in example sentences, and raise their consciousness in the process of sentence construction.

\subsection{Deficiency in the examples in LDOCE4}

Of the nineteen examples from LDOCE4 presented in Table 4, fifteen are grouped according to their contextual meanings and four are placed separately in a specific grammar box to indicate the syntactic constraints. Examples $1 c$ and $l g$ are superfluous, as they could be meant to illustrate different use of before rather than suggest. This phenomenon can also be seen in example $1 a$, in which keep doing is presented as saliently as the collocation suggest ways. Examples $1 \mathrm{~h}$ and $6 a$ are more suitable for text reception than text-production. Example $1 d$ in LDOCE4 conflicts with example $1 e$ in $O A L D 7$ in terms of the verb form in that-clause. The examples in grammar box distinctively emphasize the wrong syntactic structure suggest $s b$ to do without attaching sufficient attention to the verb form used in that-clause. However, Chinese learners tend to make errors with verb forms in that-clause as shown in the above analysis. For instance, they can produce sentences like *He suggested that we went out for walking.

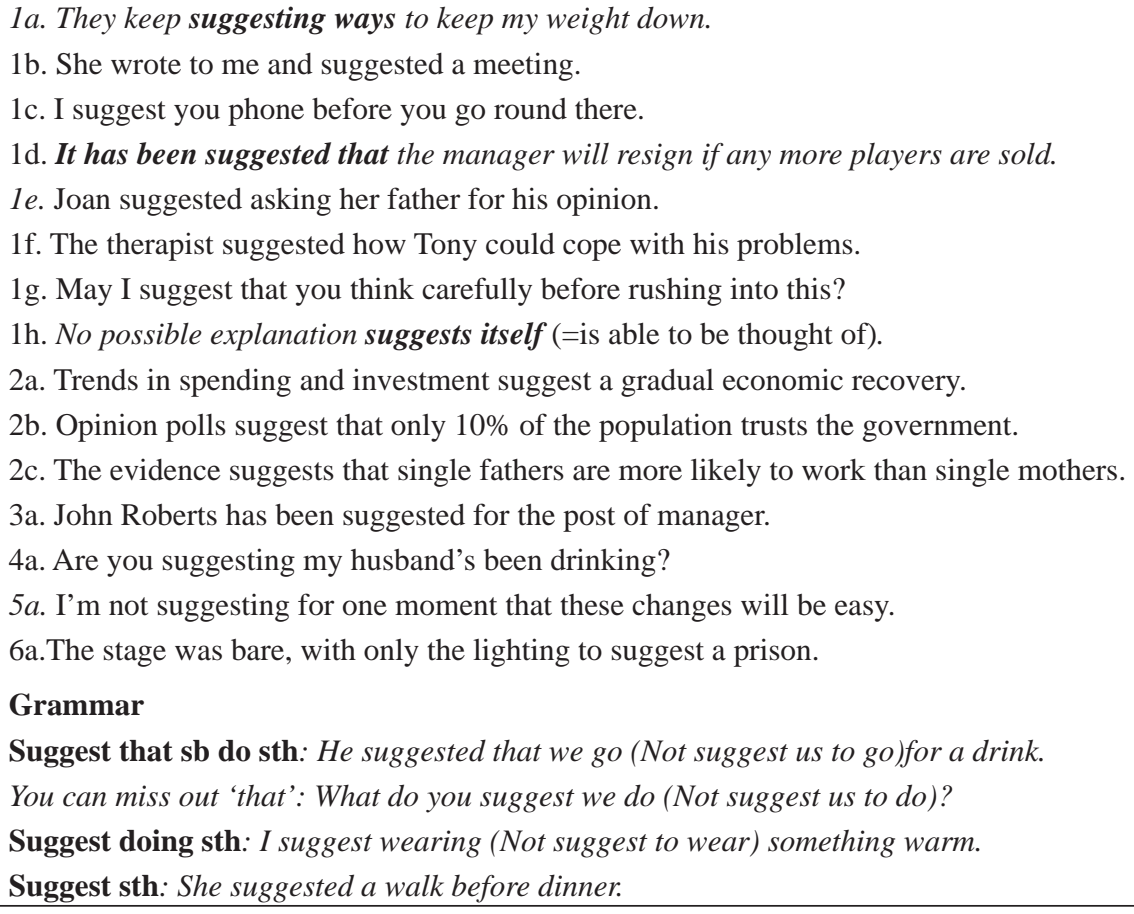

Table 4. Examples from LDOCE4

\subsection{Deficiency in the examples in LLA2}

All the examples illustrating the use of suggest in $L L A 2$ are grouped under the only one definition of the headword: "to tell someone your idea about what they should do, where they should go etc, or about what you and they should do together". These examples concentrate on providing optional ways for learners to express their ideas, when suggest takes the above-mentioned meaning. Such practice reflects the assumption that the users possess considerable grammatical knowledge about the proper syntactic structures of suggest. In this respect Humblé (2001: 69) points out that "it is not the task of dictionaries to teach grammar in a comprehensive way, but the dictionary is the place where learners look for information on particular grammar items such as syntactic constraints". Moreover, if the dictionary users want to figure out whether in certain productive contexts suggest could mean indicate, they will be discouraged. 
1. "Why don't you come with us?" Alan suggested.

2. It was a sunny afternoon, and Jim suggested a trip to the beach.

3. My Dad suggested that I should apply for the job.

4. I suggest we take a break and finish this later.

5. It was raining heavily, and she suggested calling a taxi.

6. Can you suggest where we might be able to get a decent meal?

Table 5. Example from $L L A 2$

In conclusion, the abstracted examples shown in Tables 3, 4 and 5 fail to indicate the syntactic structures and constraints saliently related to suggest in one way or another. Such practice is perhaps suitable in dictionaries for advanced learners of English engaged in text production, as they are confident in extracting information from lexicographic data. Or they may not need to consult dictionaries for the usage of suggest. But such practice may not work well with the Chinese learners defined in this study.

\section{Demonstrating some proposals for exemplification through model articles}

With the insights gathered from the analysis of the Chinese learners' lexicographic information needs in connection with writing English texts and the examination of the present lexicographic practice of providing examples in learners' dictionaries, it is now possible to present some examples in model articles constructed with full consideration of the Chinese learners' lexicographic information needs related to English writing. As the focus in the model articles is on exemplification, other types of lexicographic data are intentionally omitted. Some examples abstracted from the chosen dictionaries will be adapted for the purpose of demonstration.

\subsection{Explicitly demonstrating the possible syntactic structures}

As shown in CLEC, errors in syntactic structure are very salient in texts written by Chinese learners of English. Therefore, an explicit presentation of syntactic structures will act as signposts to guide Chinese learners to select the proper sentence structures, and raise their awareness to avoid possible errors. Moreover, typographic highlighting added to the syntactic structure encoded in sentence examples could speed up the learners' access to the relevant data. The syntactic structures as well as the sentence examples as demonstrated in Model Article 1 suggest are designed to help Chinese learners avoid their common syntactic errors. The syntactic structure presented before the sentence examples could either function independently or in combination with the sentence examples, depending on the profile of the targeted learners. A partial or complete presentation of the syntactic structure with sentence examples could be achieved in Internet-based dictionaries by hiding unnecessary data according to learners' individualized contexts. The sentence examples after suggest + (that) clearly show the typical verb forms in the sub-clause in the active as well as passive voice in order to raise the learners' awareness.

suggest /.../

1 to tell someone your ideas about what they should do, where they should go

$\Rightarrow$ propose

suggest sth: May I suggest a white wine with this dish, sir?

suggest + (that): He suggested we (should) go out to eat. // He suggested that the lecture (should) be

given in English.

suggest + -ing: I suggested going in my car.

It has been suggested + that: It has been suggested that bright children (should) take their exams early.

(Model Article 1) 


\subsection{Saliently indicating the typical patterns of syntactic behavior}

Indicating the typical patterns of syntactic behavior of the lemma has been advocated in many studies on exemplification in dictionaries (Laufer 1992; Rundell 1999; Atkins/Rundell 2008). The concept of being typical has always been intangible and ambiguous. The present study proposes that the typical syntactic behavior of a word could be its universal usage in contexts as well as the possible constraints over the contextual usage of this word. For instance, the typical syntactic behavior of the word cattle is reflected in the plural verb form following cattle in syntactic environments. Hence, examples in the article cattle are expected to display such idiosyncrasy either for ready use by foreign learners or to raise the awareness of foreign learners. The latter is especially for those learners, (e.g. Chinese learners of English) whose conceptual understanding of nominal countability differs from that of native speakers, or whose L1 lack grammatical systems corresponding to the L2 in question. It should be noted that the typical patterns of syntactic behavior of headwords should be understood from the foreign learners' perspective. For instance, some word combinations may seem to be natural for native speakers or other group of foreign learners, but they may challenge Chinese learners. For instance, Chinese learners tend to produce structures like *drink soup, *eat medicine, and *join examination, which are plainly unidiomatic for native speakers of English. Constraints on the contextual usage of certain words could be demonstrated in examples as error warnings to raise the learners' awareness in text production. Moreover, typographic highlights attached to data showing the syntactic idiosyncrasy of words will also help the learners identify the relevant data easily.

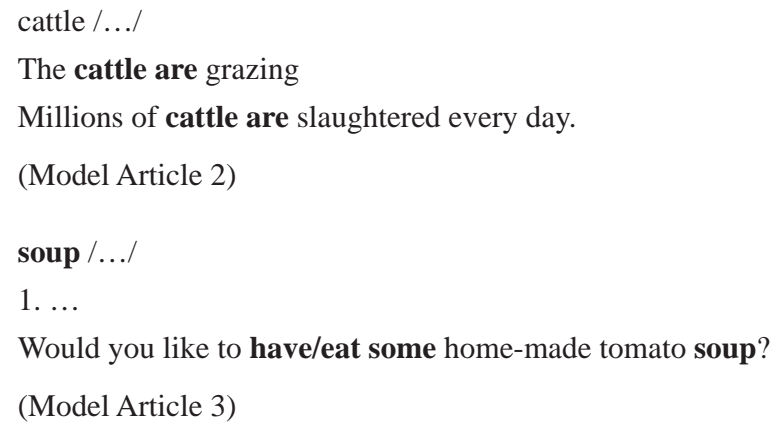

\subsection{Presenting cultural and pragmatic information}

Foreign learners engaged in text production also have needs for cultural and pragmatic information (Kachru/Kahanej 1995; Burkhanov 2003; Tarp 2008). Exemplification could act as an effective way to convey cultural and pragmatic information. In order to articulate their ideas appropriately in the target culture, Chinese learners need information about the target culture. However, one should be aware that the Chinese learners' understanding of the world is inevitably shaped by their native culture. Therefore, Chinese learners may also have lexicographic needs for information related to their native culture, for instance, the lexical items related to Chinese culture. An example like the one shown in Model Article 4 (calendar) can help Chinese learners of English express a concept related to Chinese culture and transmit information about Chinese culture simultaneously. The examples illustrating the use of forms of address, presented in Model Article 5 (dear) also offer the pragmatic information to help Chinese learners in the production of English texts.

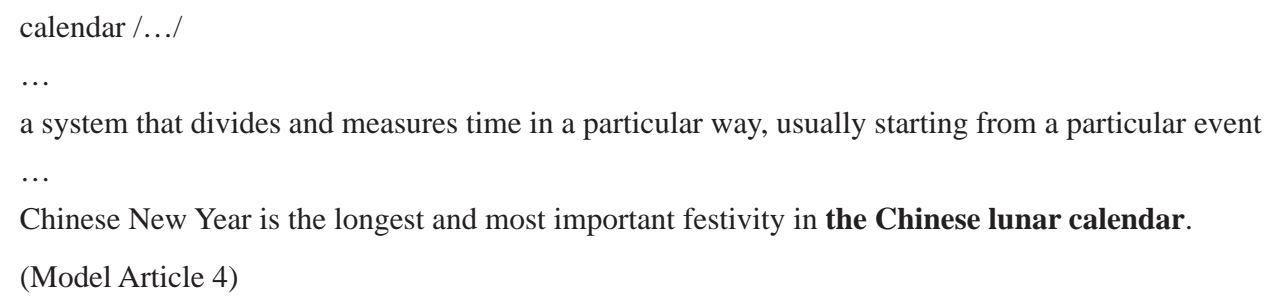


dear $/ . . . /$

used in front of someone's name at the beginning of a letter to them

Dear Mr. / Mrs./MS. /Miss + surname,

Dear Sir or Madam,

Dear+ first name,

Not: Đear teacher

(Model Article 5)

\subsection{Presenting error warnings}

As mentioned earlier, information about syntactic constraints could prevent foreign language learners from making errors in text production. There are idiosyncratic constraints concerning individual words. This study argues that more focus should be put on those constraints that tend to confuse the specific group of learners in their specific situations that involve text production. Information about usage constraints could be presented specifically in a grammar box as shown in LDOCE4, or highlighted in examples as shown in Model Article 1. It should be noted that the contents of error warnings should not be interpreted in abstract terms, that is, without reference to the profile of a specific group of learners. For instance, Chinese learners tend to produce errors like *join (an) examination instead of anite an examination marked in OALD7. The following model articles attempt to suggest error warnings through examples. For instance, due to cultural differences, Chinese learners may produce structures like *red tea instead of black tea, *black bread rather than brown bread. The error warning inserted in example in Model Article 6 could raise the Chinese learners' linguistic awareness and help them avoid such errors in text production. The warnings in Model Article 7 are intended to remind Chinese learners of their high-frequency errors with nominal countability as well as the use of article use. Depending on the profile of the learners and the quality of the errors in question, the error warnings can be presented in different ways.

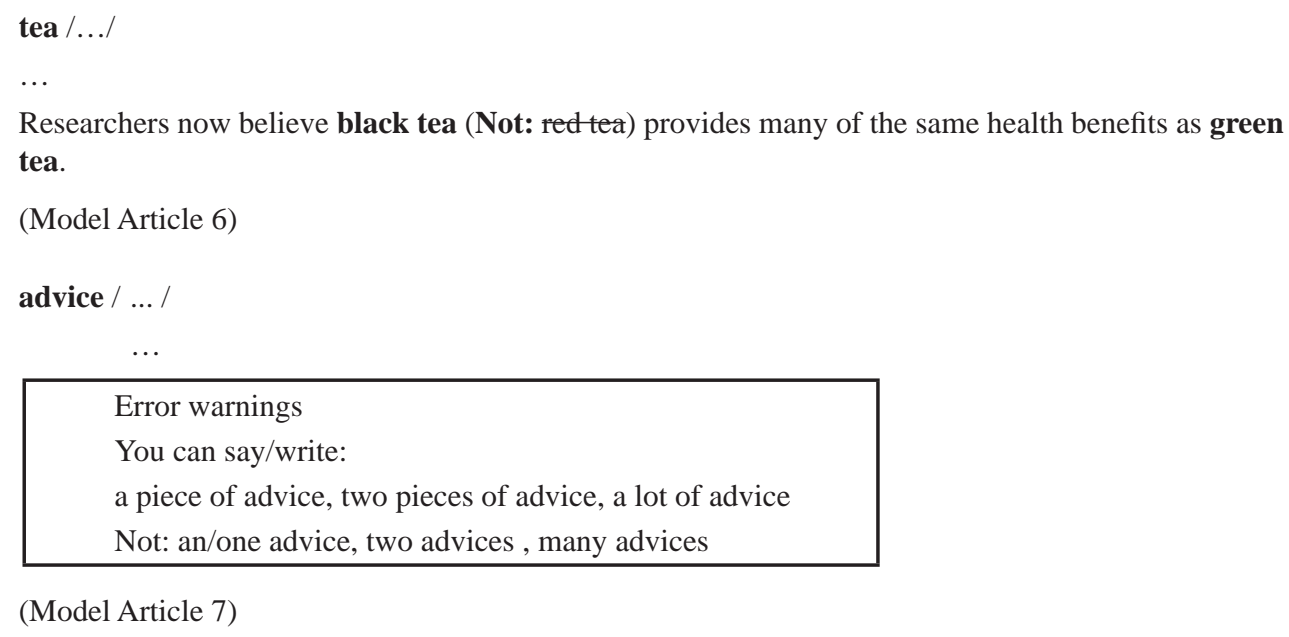

Besides, examples can confirm the correct choice of words. Admittedly, dictionary definition plays the central role in the elicitation of meaning. However, examples may help foreign learners seize the semantic subtlety of the word in question, and accordingly help them articulate their ideas appropriately. It should be noted that examples are only supplementary to definition, especially in the case of synonyms. For instance, Chinese learners tend to be confused about the use of the words efficient and effective. Example sentences like The city has a highly efficient public transportation system and This medicine is effective for coughing would be helpful. 


\section{Conclusion}

In the present study, the functional approach to lexicography has been adopted to examine the present lexicographic practice of exemplification in some learners' dictionaries and to explore how to optimize dictionary examples so that they can help Chinese learners write English texts more effectively. The study clearly demonstrates that examples are useless unless they satisfy the targeted users' lexicographic needs arising in their particular user situations. Therefore, the starting point for the discussion of exemplification in dictionaries should be the foreign learners' particular needs arising in their concrete learning activities. It is pointless to examine various types of linguistic data contained in examples, independently of the target learners' needs. The same applies to the argument over authentic or made-up examples in dictionaries. The findings of this study could be either adapted or extended to other groups of foreign language learners in the same language learning situations. However, more empirical research may be necessary to understand different groups of foreign learners' universal as well as individualized information needs in their particular situations. Given the limited space, this study is confined to printed dictionaries, so further research is also advocated to explore the optimized ways of presenting examples in online dictionaries.

\section{References}

Atkins, B.T. Sue/Rundell, Michael 2008: The Oxford Guide to Practical Lexicography. Oxford: Oxford University Press.

Bergenholtz, Henning/Tarp, Sven 2003: Two opposing theories: On H.E. Wiegand’s recent discovery of lexicographic functions. In Hermes 31, 171-196.

Burkhanov, Igor 2003: Pragmatic specifications: usage indications, labels, examples; dictionaries of styles, dictionaries of collocations. In Van Sterkenburg Piet (ed.), A practical guide to lexicography. Amsterdam/Philadephia: John Benjamins, 102-113.

Chan, Alice.Y.W. 2004: Syntactic transfer: evidence from the interlanguage of Hong Kong Chinese ESL learners. In The Modern Language Journal 88/1, 56-74.

Cortazzi, Martin/Jin, Lixian 1996: State of the Art: English Teaching and Learning in China. In Language Teaching 29, 61-80.

Cowie, Anthony Paul 1989: The language of examples in English learner's dictionaries. In James, Gregory (ed.), Lexicographers and their Works. Exeter: University of Exeter Press, 55-65.

Creamer, Thomas 1987: Beyond the definition: some problems with examples in recent Chinese-English and EnglishChinese bilingual dictionaries. In Cowie, Anthony P. (ed.), The Dictionary and the Language Learner. Tübingen: Max Niemeyer Verlag, 238-245.

Drysdale, P. D. 1987: The role of examples in a learners' dictionaries. In Cowie, Anthony P. (ed.), The Dictionary and the Language Learner. Tübingen: Max Niemeyer Verlag, 21-223.

Gui, Shi Chung/Yang, Hui Zhong 2003: Chinese Learner English Corpus. Shanghai: Shanghai Foreign Language Education Press.

Hausmann, Franz Josef/Gorbahn, Adeline 1989: COBUILD and LDOCE II: A Comparative review. In International Journal of Lexicography 2/1, 44-56.

Hu, Guang Wei 2005: English Language Education in China: Policies, Progress, and Problems. In Language Policy 4, 5-24.

Huang, Xiao Yu. 2005: Multilevel analysis of Chinese EFL learners' errors in their writing. In CELEA Journal 28(5), 24-32.

Humblé, Philippe 2001: Dictionaries and Language Learners. Frankfurt am Main: Haag und Herchen.

Humblé, Philippe 1998: The Use of Authentic, Made-up and 'Controlled' Examples in Foreign Language Dictionaries. In Fontenelle et al. (eds.), EURALEX '98 Proceedings, 543-599.

Jin,Yan/Yang, Hui Zhong 2006: The English Proficiency of College and University Students in China: As Reflected in the CET. In Language, Culture and Curriculum 19/1, 21-36.

Kachru, B. Braj/Kahanej, Henry (eds) 1995: Cultures, Ideologies, and the Dictionary: Studies in Honor of Ladislav Zgusta. Tubingen: Niemeyer. 
Laufer, Batia 1992: Corpus-based versus lexicographer examples in comprehension and production of new words. In H. Tommola et al. (eds.), EURALEX'92 Proceedings, 71-76.

Mohan, A. Bermard /Lo, A.Y. Winnie 1985: Academic writing and Chinese students: Transfer and developmental factors. In TESOL Quarterly 19: 515-534.

Nesi, Hilary 2000: The Use and Abuse of EFL Dictionaries. Tübingen: Max Niemeyer Verlag.

Rundell, Michael 1999: Dictionary use in Production. In International Journal of Lexicography 12/1, 35-53.

Summers, Della 2003: Longman Dictionary of Contemporary English (4th Edition.). London: Pearson Education Ltd. Summers, Della 2002: Longman Dictionary of Contemporary English (2nd Edition.). London: Pearson Education Ltd. Tang, Qi Ming 2006: Lexical Mismatching in Chinese Students’ EFL Writing. In Sino-US English Teaching 3.5, 10-16.

Tarp, Sven 2008: Lexicography in the Borderland between Knowledge and Non-Knowledge. Tubingen: Max Niemeyer Verlag.

Wang, Wen Yu/Wen, Qiu Fang 2002: L1 use in the L2 composing process: An exploratory study of 16 Chinese EFL writers. In Journal of Second Language Writing 11, 225-246.

Wehmeimer, Sally (eds.) 2005: Oxford Advanced Learners' dictionaries of Current English (7 $7^{\text {th }}$ edition.). London: Oxford University Press.

Wu, Yi’an 2001: English language teaching in China: Trends and challenges. In TESOL Quarterly 35/1, 191-194.

Xu, Hai 2008: Exemplification Policy in English Learners’ Dictionaries. In International Journal of Lexicography 21/4, 395-417.

Xu, Hai 2006: Investigating Users' Needs for Illustrative Examples: the Case of Chinese EFL learners. In Cadernos de tradução 2, 1-22.

Yang,Wen Xiu 2002: Pragmatic competence, linguistic competence, communicative competence. In Foreign languages and Their teaching 3, 5-8.

Yang, Yi 2001: Chinese Interference in English Writing: Cultural and Linguistic Differences [online]. http://eric.ed.gov/ ERICDocs/data/ericdocs2sql/content_storage_01/0000019b/80 /19/cf/d0.pdf. (Accessed 9 May 2009). 